\title{
Trends in childhood leukaemia in the Nordic countries in relation to fallout from atmospheric nuclear weapons testing
}

\author{
Sarah C Darby, Jørgen H Olsen, Richard Doll, Bharat Thakrar, Peter de Nully Brown, \\ Hans H Storm, Lotti Barlow, Frøydis Langmark, Lyly Teppo, Hrafn Tulinius
}

Imperial Cancer Research

Fund Cancer Epidemiology Unit, University of Oxford,

Radcliffe Infirmary, Oxford

OX2 6HE, United

Kingdom

Sarah C Darby, staff scientist

Richard Doll, honorary

consultant

Bharat Thakrar, research

officer

Danish Cancer Society,

Danish Cancer Registry,

DK-2100, Copenhagen $\emptyset$,

Denmark

Jørgen H Olsen, section chief

Peter de Nully Brown, staff

scientist

Hans H Storm, section chief

Swedish Cancer Registry, National Board of Health and Welfare, S-10630

Stockholm, Sweden

Lotti Barlow, assistant director

\section{Cancer Registry of}

Norway-Institute for

Epidemiological Cancer

Research, Montebello,

Oslo 3, Norway

Frøydis Langmark, director

Finnish Cancer Registry-

Institute for Statistical and

Epidemiological Cancer

Research, Liisankatu 21B,

SF-00170 Helsinki, Finland

Lyly Teppo, director

Icelandic Cancer Registry, PO Box 5420, IS-125

Reykjavik, Iceland

Hrafn Tulinius, professor

Correspondence to:

Dr Darby.

BM7 1992;304:1005-9

\section{Abstract}

Objective-To obtain further information about the risks of childhood leukaemia after exposure to ionising radiation at low doses and low dose rates before or after birth or to the father's testes shortly before conception.

Design-Observational study of trends in incidence of childhood leukaemia in relation to estimated radiation exposures due to fallout from atmospheric nuclear weapons testing during the 1950 s and 1960s.

Setting-Nordic countries.

Subjects-Children aged under 15 years.

Main outcome measures-Incidence rates of leukaemia by age at diagnosis, sex, country, and calendar year of diagnosis or year of birth; exposure category; relation between leukaemia and exposure for children aged 0-14 and 0-4 separately.

Results-During the high fallout period the average estimated dose equivalent to the fetal red bone marrow was around $140 \mu \mathrm{Sv}$ and the average annual testicular dose $140 \mu \mathrm{Sv}$. There was little evidence of increased incidence of leukaemia among children born in these years. Doses to the red bone marrow of a child after birth were higher, and during the high exposure period children would have been subjected to an additional dose equivalent of around $1500 \mu \mathrm{Sv}$, similar to doses received by children in several parts of central and eastern Europe owing to the Chernobyl accident and about $50 \%$ greater than the annual dose equivalent to the red bone marrow of a child from natural radiation. Leukaemia incidence and red marrow dose was not related overall, but rates of leukaemia in the high exposure period were slightly higher than in the surrounding medium exposure period (relative risk for ages $0-14: 1.07$, $95 \%$ confidence interval 1.00 to 1.14 ; for ages $0-4$ : $1 \cdot 11,1.00$ to $1 \cdot 24)$.

Conclusions-Current predicted risks of childhood leukaemia after exposure to radiation are not greatly underestimated for low dose rate exposures.

\section{Introduction}

Evidence for the magnitude of the risk of childhood leukaemia after childhood exposure to ionising radiation delivered at a high dose rate is substantial from studies of the Japanese survivors of the atomic bomb, ${ }^{1}$ infants given radiotherapy for supposed thymus enlargement, ${ }^{2}$ and children irradiated for tinea capitis. ${ }^{3}$ Studies of the effects of diagnostic $x$ rays in pregnancy also provide good quantitative evidence of the magnitude of the risk to the fetus after high dose rate exposures. ${ }^{45}$

In contrast, there is still considerable uncertainty about the magnitude of the risk after exposures to low doses of ionising radiation delivered at low dose rates.
Reports of an increased incidence of childhood leukaemia in the vicinity of the two nuclear installations in Britain in which nuclear fuel is reprocessed ${ }^{67}$ have given rise to concern about the possible effects of radioactive pollution of the environment, concern which has been greatly exacerbated by knowledge of the pollution caused by the nuclear accident at Chernobyl. Estimates of the risks involved ${ }^{8}$ have suggested that the amounts of waste dispersed in western Europe would not produce any detectable effect, not even as a result of the accident at Chernobyl. ${ }^{9}$ These estimates, however, are based on the effects of high dose rate exposure, and they have not, hitherto, taken account of the possibility suggested by Gardner et al that irradiation of the testes may cause an increased risk of leukaemia in any offspring subsequently conceived within a few months. ${ }^{10}$ It therefore seems important to test the validity of the estimates by direct observation of the effects of the waste. Such an opportunity is provided by examining the trends in the incidence of childhood leukaemia around the time when nuclear weapons were being tested intensively in the atmosphere, as the pollution which has given rise to concern is similar in character to that produced by weapons testing.

An early attempt to use trends in mortality from leukaemia in childhood and in the incidence of leukaemia in England and Wales was complicated by temporal changes in the efficacy of treatment and in the efficiency of cancer registration,,$^{11}$ and we can hope to obtain useful information only from data on incidence of leukaemia recorded by cancer registries that were already operating before 1962 . We have, therefore, sought evidence from the cancer registries in Denmark, Finland, Iceland, Norway, and Sweden, which together cover a large enough population (about 20 million) to provide figures subject to proportionately small random error, which have long obtained practically complete registration, and which cover countries where radioactive fallout from weapons testing was relatively high.

Methods

EXPOSURE TO RADIATION FROM WEAPONS FALLOUT

Estimates of annual doses to the red bone marrow or testes from weapons fallout have not been published for any of the Nordic countries. Estimates of the dose equivalent to the red bone marrow for a fetus and for a 1 year old child in Britain have, however, been published (see table I). ${ }^{12}$ The dose equivalent is obtained by summing the absorbed dose from low linear energy transfer radiation ( $x$ rays and $\gamma$. rays) and 20 times the absorbed dose from high linear energy transfer radiation (neutrons and $\alpha$ particles). The dose equivalent to the red bone marrow of a 10 year old child followed a similar pattern to that experienced by a 1 
year old child and took about two thirds the value. Doses to the testis from weapons fallout in Britain have not been published but were estimated here by combining the annual effective dose equivalent to an adult through external radiation, which has been published, ${ }^{12}$ with the annual internal dose from caesium-137 calculated by $\mathrm{T} P$ Fell (personal communication) (see table I). Doses from weapons fallout in north west Europe depend on latitude, rainfall, and dietary habits, and so doses received in the Nordic countries may differ in magnitude from those in Britain. However, the pattern of variation with calendar time should be similar in all six countries. The British data were therefore used to classify the dose received each year during the period of fallout as low, medium, or high (table I).

\section{LEUKAEMIA REGISTRATION DATA}

Observed numbers of leukaemias for boys and girls were collated for single years of age together with the corresponding estimated populations. The data were classified both by calendar year of birth and by calendar year of diagnosis in single years. For each registry, data were obtained from the start of operations. There is, however, inevitably some doubt about the completeness of registration in the initial years, and so data for the first five years from the three registries that started earliest (Denmark, Finland, and Norway) were excluded, and data for the first three years from Iceland and Sweden were excluded. Consequently, data were included from 1948 for Denmark; from 1958 for Finland, Norway, and Iceland; and from 1961 for Sweden. For each registry data were assumed to be complete up to the end of 1987. (Copies of the basic tabular data are available from B Thakrar.)

\section{ANALYSIS}

The observed numbers of leukaemia registrations were assumed to have a Poisson distribution with a mean proportional to the associated population and depending on country, sex, age, and either individual year of diagnosis or individual year of birth. Models

TABLE I-Estimated dose equivalents $(\mu S v)$ from weapons fallout to red bone marrow for fetus or 1 year old child and to testes of adult in Britain by calendar year and classification of exposure.

\begin{tabular}{|c|c|c|c|c|c|}
\hline Year & Fetus & $\begin{array}{l}1 \text { year old } \\
\text { child }\end{array}$ & $\begin{array}{l}\text { Classification of } \\
\text { calendar years for } \\
\text { bone marrow } \\
\text { dose equivalents }\end{array}$ & $\begin{array}{c}\text { Testes of } \\
\text { adult }\end{array}$ & $\begin{array}{l}\text { Classification of } \\
\text { calendar years } \\
\text { for testis dose } \\
\text { equivalents }\end{array}$ \\
\hline 1951 & 5 & 12 & & 8 & \\
\hline 1952 & 5 & 16 & & 8 & Low-1 \\
\hline 1953 & 11 & 36 & ILW-1 & 19 & \\
\hline 1954 & 9 & 29 & Low-1 & 16 & \\
\hline 1955 & 12 & 54 & & 20 & \\
\hline 1956 & 15 & 63 & & 21 & \\
\hline 1957 & 22 & 82 & & 31 & \\
\hline 1958 & 32 & 140 & & 42 & Medium-1 \\
\hline 1959 & 41 & 180 & Medium-1 & 48 & \\
\hline 1960 & 19 & 90 & & 19 & \\
\hline 1961 & 22 & 110 & & 21 & \\
\hline 1962 & 54 & 220 & & 56 & \\
\hline 1963 & 90 & 420 & High & 89 & High \\
\hline 1964 & 80 & 390 & & 76 & \\
\hline 1965 & 56 & 260 & & 55 & \\
\hline 1966 & 35 & 170 & & 29 & Medium-2 \\
\hline 1967 & 23 & 120 & & 14 & \\
\hline 1968 & 21 & 100 & Medium-2 & 11 & \\
\hline 1969 & 19 & 91 & & 10 & \\
\hline 1970 & 19 & 85 & & 11 & \\
\hline 1971 & 18 & 80 & & 12 & \\
\hline 1972 & 15 & 64 & & 9 & \\
\hline 1973 & 13 & 57 & & 7 & Low-2 \\
\hline 1974 & 12 & 49 & & 7 & \\
\hline 1975 & 11 & 42 & & 6 & \\
\hline 1976 & 10 & 35 & Low-2 & 5 & \\
\hline 1977 & 10 & 38 & & 6 & \\
\hline 1978 & 10 & 35 & & 6 & \\
\hline 1979 & 9 & 39 & & 5 & \\
\hline
\end{tabular}

Exposure for 1 year old child was considered low in any calendar year for dose equivalent in Britain $<75 \mu \mathrm{Sv}$, medium 75-200 $\mu \mathrm{Sv}$, and high $\geqslant 200$ dose equivalent in Britain $<75 \mu \mathrm{Sv}$, medium $75-200 \mu \mathrm{Sv}$, and high $\geqslant 200$ $\mu \mathrm{Sv}$ and for fetus and adult testes low for dose equivalent $<18 \mu \mathrm{Sv}$, mordic countries were about double those in Britain.

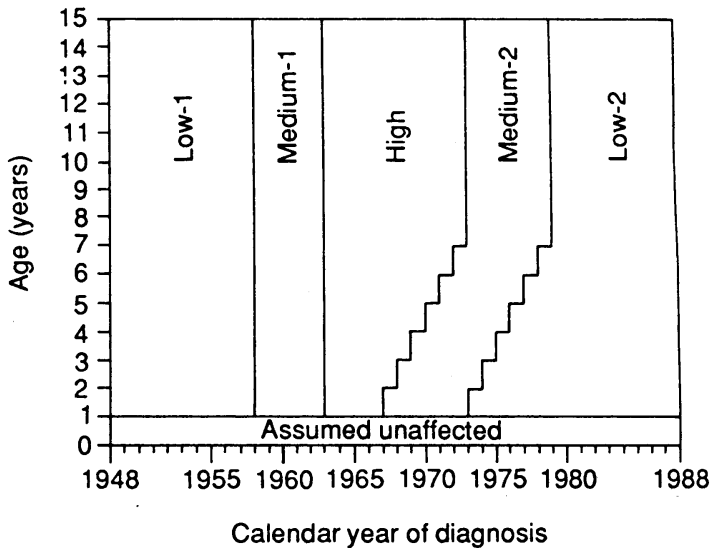

FIG 1-Allocation of age and calendar year specific leukaemia rates to exposure categories for examining effect of irradiation received after birth (for example, leukaemia registrations in children aged between 9 and 10 years occurring during 1970 are allocated to high exposure category; registrations occurring in same year in children aged 2-3 years are allocated to medium-2 exposure category, as these children were born after years when annual dose equivalent to red bone marrow of child was classified as high, see table I). Children aged $<1$ year are assumed to be unaffected by radiation received afier birth and are excluded

which included these factors as multiplicative terms were fitted to the data using the GLIM computer program. ${ }^{13}$ Estimated relative risks were based on these models. Plotted rates for individual years of diagnosis or years of birth were the arithmetic means of the resulting fitted rates for boys and girls at each year of age in each of the five countries. There was no evidence of variation beyond that due to the Poisson distribution, as assessed by comparing the residual deviances with their appropriate numbers of degrees of freedom.

The possible effects of irradiation from fallout received $(a)$ after birth, $(b)$ in utero, or $(c)$ to the father's testes were examined by fitting a further series of models in which the observed numbers of leukaemia registrations were assumed to depend on their associated populations and also on country, sex, age, and exposure category. Exposure category was derived from the data given in table I in several ways. Firstly, for radiation received after birth the data for age and calendar year of diagnosis were grouped into exposure categories (fig 1). This grouping was obtained by considering the classification of calendar years for the dose equivalent to the bone marrow of a 1 year old child, shown in table I, and assuming that red bone marrow exposure in a given calendar year could affect only children born before the year in question; that children aged under 1 year would not be affected; and that for older children the effect of exposure in any given year would be apparent in the calendar year after the year in which the exposure occurred and would last for seven years. This period was chosen with reference to studies of individuals exposed to high doses of radiation, such as patients with ankylosing spondylitis treated with $x$ rays, ${ }^{14}$ in whom the risk of radiation induced leukaemia seems greatest from one to seven years after exposure. For each age specific and calendar year specific group the data were allocated to the high exposure category if subjects were found to be affected by fallout received in one or more high exposure years according to the rules given above and to the medium exposure category if they were found to be affected by fallout from at least one medium exposure year but no high exposure years. Secondly, for radiation received by the fetus the relevant exposure at every age was assumed to be the estimated bone marrow dose to the fetus during the year of birth. The data on age and calendar year of birth were therefore grouped into exposure categories by considering the classification of calendar years for the fetal bone marrow dose (table I) during the relevant year of birth. Thirdly, for radiation 
to the father's testes the relevant dose was assumed to be the estimated testicular dose during the year before the year in which the child was born. The data for age and calendar year of birth were therefore grouped into exposure categories by considering the classification of calendar years for the dose to the testis (table I) during the year before the relevant year of birth.

In each model the significance of the various factors was assessed by omitting the factor from the model and comparing the increase in residual deviance with the appropriate $\chi^{2}$ distribution. Confidence intervals were calculated from the estimated log relative risks and their estimated standard errors.

It was decided a priori to carry out all analyses for subjects aged 0-14 combined and also for those aged $0-4$ separately as the reported increases in childhood leukaemia in the vicinity of nuclear installations are concentrated in the younger age group.

\section{Results}

\section{CALENDAR YEAR OF DIAGNOSIS}

Table II shows the estimates of the relative risks obtained when the data were classified by country, sex, age, and calendar year of diagnosis and when ages 0-14 years were included in the analysis, and figure 2 shows the corresponding rates of leukaemia by calendar year of diagnosis. As expected, the incidence rate at ages under 15 years in girls was significantly lower than in boys (relative risk $0.83 ; p<0.001$ ). There were also significant differences in the relative risks by age at diagnosis $(p<0.001$ for heterogeneity), and they showed the characteristic pattern associated with childhood leukaemia - that is, an increase in incidence to a peak at ages 2-3 and then a steady decrease until at ages 9-14 the rate was only about two thirds of that experienced in the first year of life. Relative risks showed little variation between the five Nordic countries ( $p=0.22$ for heterogeneity). Data for the calendar years before 1958 were available for Denmark alone, and so the rates during this period show considerable random variation (fig 2). During 1958-79 the rates remained roughly constant, although the highest values occurred during three consecutive years 1967-9, which correspond roughly to the years when any effect of fallout dose to the red bone marrow of the child would be at a maximum. After 1979 there is some suggestion that the rates may be increasing.

When the analysis was repeated including only children aged under 5 years at diagnosis the relative risks by sex, age at diagnosis, and country were similar to those obtained with data on children at ages 0-14. Figure 3 shows the corresponding rates of leukaemia by calendar year. As before, greater variability was apparent before 1958, when only Danish data were available, and there was again some increase after 1979. During 1958-79 the rate seemed to rise to a peak in about 1967 and then to fall. The years surrounding 1967 correspond to the period when any effect of fallout dose to the red bone marrow of the child would be at a maximum.

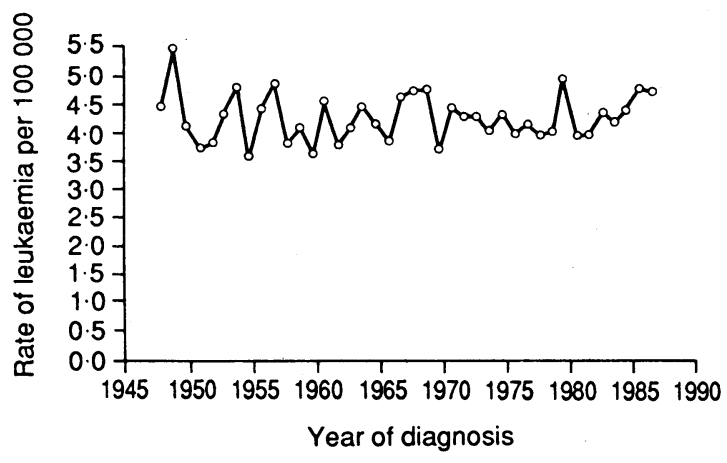

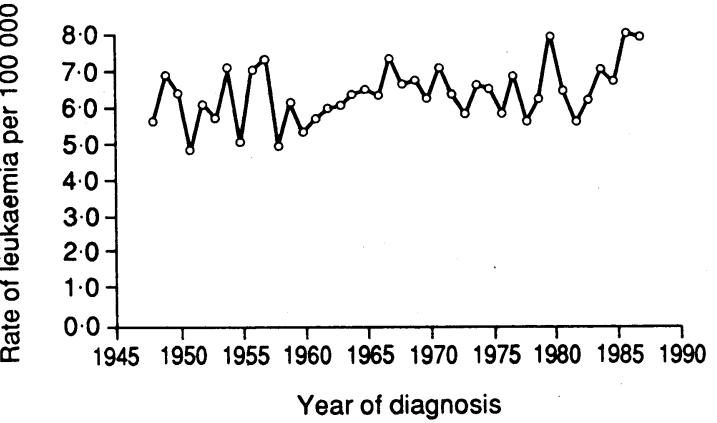

FIG 3-Rates of childhood leukaemia per 100000 at ages 0-4 by calendar year of diagnosis in Nordic countries, 1948-87, standardised for age, sex, and country

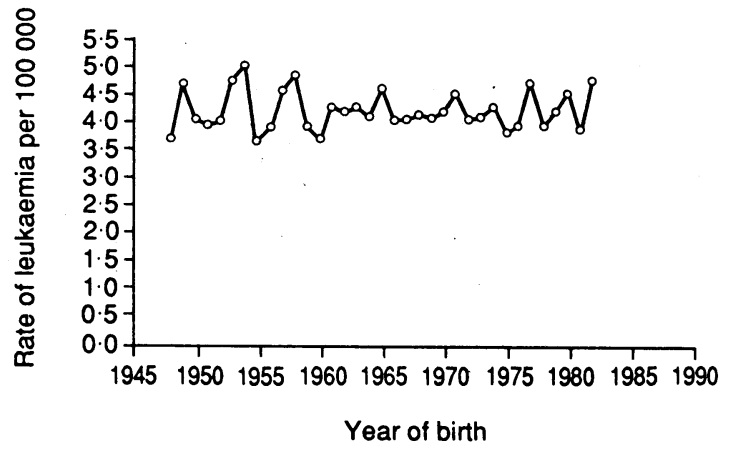

FIG 4-Rates of childhood leukaemia per 100000 at ages 0-14 by calendar year of birth in Nordic countries, 1948-82, standardised for age, sex, and country

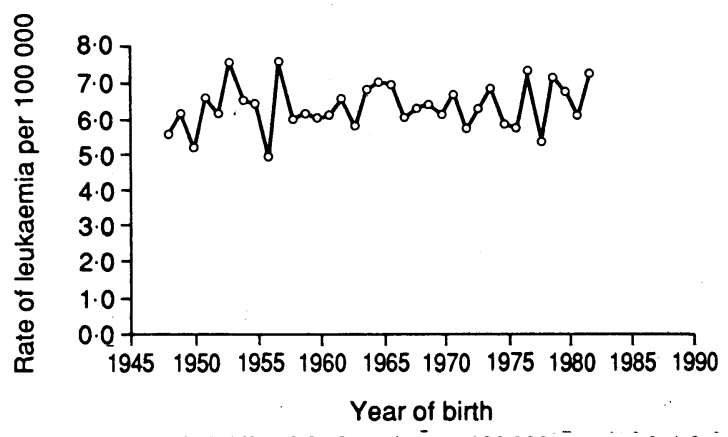

FIG 5-Rates of childhood leukaemia per 100000 at ages $0-4$ by calendar year of birth in Nordic countries, 1948-82, standardised for age, sex, and country

\section{YEAR OF BIRTH}

When the data were classified according to year of birth rather than calendar year of diagnosis the relative risks by sex, age at diagnosis, and country remained very similar to those given in table II. Figure 4 shows the corresponding rates by calendar year of birth for ages 0 -14 and figure 5 for ages 0-4. Increased variability was again apparent before 1958, when only Danish data were available. For children born in subsequent years no overall trends were apparent. When ages $0-14$ were considered high rates of leukaemia were observed for children born in 1965, 1971, 1977, and 1982 (fig 4), and when ages 0-4 were considered high rates were observed for children born in 1964-6, 1971, 1974, 1977, and 1979 (fig 5). The period 1964-6 does not quite correspond with the period when any effect of fallout dose to the red bone marrow of the fetus or to the father's testes in the year before birth would be at a maximum, especially when considered in conjunction with the low rate of leukaemia observed in 1963.

\section{EXPOSURE CATEGORIES BASED ON IRRADIATION OF RED} BONE MARROW AFTER BIRTH

When data for calendar year of diagnosis were grouped into exposure categories based on irradiation of the red bone marrow after birth and ages 0-14 were 
considered the relative risks in the five exposure categories were not significantly different $(p=0.22$ for heterogeneity). The relative risk in the high exposure category was similar to that in the low exposure categories and slightly higher than in the medium exposure categories (table III). When compared directly with that in the two medium exposure categories combined, the relative risk in the high exposure category was 1.07 ( $95 \%$ confidence interval 1.00 to $1 \cdot 14)$.

TABLE III-Relative risk of childhood leukaemia for categories of exposure to weapons fallout derived by allocating data into exposure categories based on dose equivalent to red bone marrow of child after birth, or dose equivalent to red bone marrow received by fetus, or father's testicular dose in year before birth. Values are standardised for age, sex, and country

\begin{tabular}{|c|c|c|c|c|c|}
\hline & \multicolumn{5}{|c|}{ Exposure category } \\
\hline & Low-1* & Medium-1 & High & Medium-2 & Low-2 \\
\hline \multicolumn{6}{|c|}{ Dose equivalent to red bone marrow after birth } \\
\hline Ages 0-14 & 1.00 & 0.92 & 1.01 & 0.96 & 1.00 \\
\hline Ages 0-4 & 1.00 & 0.91 & $1 \cdot 10$ & $1 \cdot 04$ & $1 \cdot 10$ \\
\hline \multicolumn{6}{|c|}{ Dose equivalent to red bone marrow of fetus } \\
\hline Ages 0-14 & 1.00 & 1.01 & 1.03 & 1.00 & $1 \cdot 01$ \\
\hline Ages 0-4 & 1.00 & 1.02 & $1 \cdot 08$ & 1.06 & 1.08 \\
\hline \multicolumn{6}{|c|}{ Dose equivalent to father's testes in year before birth } \\
\hline Ages 0-14 & $1 \cdot 00$ & 0.99 & 1.01 & 0.96 & $1 \cdot 00$ \\
\hline Ages 0-4 & 1.00 & 0.99 & 1.06 & 0.96 & $1 \cdot 04$ \\
\hline
\end{tabular}

When only children aged under 5 years at diagnosis were considered there was evidence of differences between the exposure categories ( $p$ value for heterogeneity 0.02 ). The rate of leukaemia was high in the high exposure category (relative risk $1 \cdot 10$ ) and also in the second low category $(1 \cdot 10)$ (table III). When compared with the two medium exposure categories combined the relative risk in the high exposure category was $1 \cdot 11(1 \cdot 00$ to $1 \cdot 24)$.

EXPOSURE CATEGORIES BASED ON IRRADIATION OF RED BONE MARROW OF FETUS

Data for calendar year of birth grouped into exposure categories defined according to the bone marrow dose from weapons fallout received by the fetus showed no significant differences between exposure categories at ages $0-14$ ( $p>0.50$ for heterogeneity; table III). When the high exposure category was compared with the two medium exposure categories combined, the relative risk was $1.03(0.95$ to 1.11$)$.

When only those aged under 5 years at diagnosis were considered there were again no significant differences between exposure categories ( $p>0.50$ for heterogeneity; table III), and when the high exposure category was compared with the two medium exposure categories combined the relative risk was again 1.03 $(0.93$ to $1 \cdot 15)$.

\section{IRRADIATION TO FATHER'S TESTES}

Allocating data by calendar year of birth to exposure categories according to the likely dose to the father's testes during the year before birth showed no significant differences for either ages $0-14$ or the restricted age group $0-4$ ( $p>0.50$ for heterogeneity in both cases; table III). When the high exposure category was compared with the two medium exposure categories combined the relative risk was $1.02(0.94$ to 1.11$)$ for ages $0-14$ and $1.08(0.96$ to 1.22$)$ for ages $0-4$.

\section{Discussion}

In this study, data from the Nordic countries have confirmed the slightly higher risk of leukaemia for boys compared with girls, and showed the characteristic age distribution of childhood leukaemias, with a peak at ages 2-3 years. When the data were classified according to the year of birth of the child, there was little evidence for an increased risk of leukaemia among children born in those years when fallout doses were highest, and similarly negative results were found when the birth cohorts were grouped into exposure categories based on estimated radiation dose to the fetus or to the father's testes. When the data were classified according to calendar year of diagnosis there were no strong overall trends but the rates of leukaemia in the later years of the study seemed to be increasing, and they were also high in the late 1960 s, when any effect of fallout dose to the red bone marrow would be at a maximum (figs 2 and 3 ). These suggestions were stronger at ages 0-4 and were confirmed when the data were grouped into exposure categories based on bone marrow dose to the child. There was evidence of heterogeneity between the exposure categories at ages $0-4$, with increased risks in the low exposure category at the end of the study and also in the high exposure category, when any effect of fallout would be at a maximum.

Organ doses from weapons fallout have not been estimated explicitly for the Nordic countries, but information is available on the levels of strontium- 90 and ${ }^{137} \mathrm{Cs}$ in milk and the total diet and on body burdens of ${ }^{137} \mathrm{Cs}$ and ${ }^{90} \mathrm{Sr}$ levels in bone in both Britain and the Nordic countries. ${ }^{15}$ This indicates that doses in Denmark are likely to have been similar to those received in the United Kingdom whereas those in Sweden, Finland, Norway, and Iceland would have been about $50 \%, 100 \%, 300 \%$, and $300 \%$ higher respectively. Overall, therefore, average doses in the Nordic countries were probably about twice those in Britain. On this basis the average dose equivalent from weapons fallout to the red bone marrow of a fetus in the Nordic countries during the years classified as high exposure is estimated at around $140 \mu \mathrm{Sv}$, and the average testicular dose to an adult in the year before conception is estimated as $140 \mu \mathrm{Sv}$ in the same years.

These values are between two and three times the doses estimated for the medium exposure years. These paternal testicular doses are much lower than those reported to be associated with an increased risk in the vicinity of Sellafield, where paternal occupational doses in the range 5000-9999 $\mu \mathrm{Sv}$ during the six months before conception were associated with a relative risk of leukaemia of 3.54 whereas doses of $10000 \mu \mathrm{Sv}$ and above were associated with a relative risk of $7 \cdot 17$. Thus the absence of any clear increase in risk associated with exposure categories defined in terms of paternal testicular dose does not necessarily contradict the suggestion of a risk near Sellafield. ${ }^{10}$

Dose equivalents from weapons fallout to the red bone marrow of a child in the Nordic countries were higher than fetal bone marrow doses or paternal testicular doses. During the four years classified as high exposure they were of the order of $600 \mu \mathrm{Sv}$ per year at age 1 year and $400 \mu S v$ at age 10 years, while for the 11 years classified as medium exposure the corresponding values were of the order of $200 \mu \mathrm{Sv}$ and $150 \mu \mathrm{Sv}$ respectively.

If any increase in risk of leukaemia is assumed to be concentrated in the period one to seven years after the exposure the dose from weapons fallout contributing to any increase in the risk for the specific age and calendar year combinations classified as high exposure would be of the order of $1500 \mu \mathrm{Sv}$ and the corresponding dose for the age and calendar year combinations classified as medium exposure would be of the order of $500 \mu \mathrm{Sv}$. Thus the difference in the fallout doses that would be contributing to risk of leukaemia in high and medium exposure categories respectively would be of the order of $1000 \mu \mathrm{Sv}$. This value is similar to the annual dose equivalent to the red bone marrow of a child from natural radiation. In contrast, the difference in fallout 
doses that would be contributing to the risk of leukaemia in medium and low exposure categories would be less than half this value.

Temporal trends in incidence rates caused by factors other than weapons fallout complicate comparisons involving periods separated by many calendar years, whose effects can be reduced by comparing periods close in calendar time that have large differences in dose, such as the high exposure category and the two medium exposure categories. At ages $0-4$ the relative risk in the high exposure category compared with the two medium exposure categories combined was $1 \cdot 11$ $(1.00$ to 1.24$)$ whereas at ages $0-14$ it was $1.07(1.00$ to $1 \cdot 14)$.

According to the recent recommendations of the United States Committee on the Biological Effects of Ionizing Radiations (BEIR V), ${ }^{8}$ which are based on analyses of the survivors of the atomic bombings at Hiroshima and Nagasaki, a bone marrow dose of $1000 \mu \mathrm{Sv}$ in early life might be expected to cause about a $3 \%$ increase in the risk of leukaemia. This value is somewhat smaller than the $7 \%$ increase seen at ages $0-14$ and the $11 \%$ increase seen at ages $0-4$, although the $95 \%$ confidence intervals for the increases in our study both include $3 \%$. Some difference must, however, be expected as the BEIR V estimates have been calculated on a different basis to those in this study, in which attention was concentrated on children in the first seven years after exposure. In contrast, the BEIR V committee assumed a uniform increase in risk for all exposures incurred in the age range 0-20 years and also for all times during five to 15 years after exposure.

High quality data on the Japanese survivors of the atomic bomb are not available for the period two to five years after exposure, which is the period for which the relative risks of leukaemia were greatest in the study of patients irradiated for ankylosing spondylitis,${ }^{14}$ and so the committee also assumed that the relative risk in this period would be the same as that observed in the period five to 15 years after exposure. Our study provides evidence that risks based on the survivors of the atomic bomb do not grossly underestimate the risks from low dose rate exposures. The present data are compatible with risks similar to or less than those predicted by the BEIR V committee, although they are also compatible with somewhat higher risks. Risks about double those predicted by the committee were found in a study of the effect of local fallout near the Nevada test site. ${ }^{16}$

Dose equivalents received during the first year after the Chernobyl accident were reviewed by the United Nations Scientific Committee on the Effects of Atomic Radiation (UNSCEAR). ${ }^{15}$ Doses in the second year after the accident are estimated to be about half those in the initial year and doses in the third year about a fifth of those in the initial year. ${ }^{17}$ If the total dose from the Chernobyl accident is assumed to be 1.7 times the estimated dose in the first year, average bone marrow dose equivalents to children living in southern Switzerland, north eastern Romania, Bulgaria, northern Greece, Austria, and the area of the Ukraine to the south west of Chernobyl would have been in the range $1000-2200 \mu \mathrm{Sv}$, and those to children living in the region immediately to the north and west of Chernobyl would have been higher. For other areas estimated marrow doses are below $1000 \mu \mathrm{Sv}$ and in most cases substantially so.

Exposures after the Chernobyl accident were primarily from ingested ${ }^{137} \mathrm{Cs}$, with some contribution from external $\gamma$ rays. They would thus have been of similar type to that received from weapons fallout. Furthermore, although the United Nations committee did not consider children specifically, the bone marrow doses received by children would have been similar to those received by adults. ${ }^{18-20}$ Thus if the increases seen in the Nordic countries in the high exposure period compared with the medium exposure periods are caused by weapons fallout and if the estimates of bone marow doses due to weapons fallout and the accident at Chernobyl are not greatly in error, then the accident would be expected to cause increases in the incidence of childhood leukaemia in the above areas with estimated doses of $1000 \mu \mathrm{Sv}$ or more that are comparable to or greater than the increases seen in our study. For all other areas the effects of the accident should be similar to or less than that of the effect of fallout from atmospheric nuclear weapons testing in the 1950s and '60s in the Nordic countries.

We thank Bendix Carstensen, Frances Fry, Heinz Hansen, Mary Morrey, Colin Muirhead, and John Stather for helpful discussions in preparing this paper, and Lindsey Cutler for help in preparing the manuscript.

1 Shimizu Y. Studies of the mortality of A-bomb survivors part 9. Mortality, 1950-1985; part 2. Cancer mortality based on the recently revised doses 1950-1985; part 2. Cancer mortality based

2 Pifer JW, Toyooka ET, Murray RW, Ames WR, Hempelmann LH. Neoplasms in children treated with $x$ rays for thymic enlargement. I. Neoplasms and mortality. I Natl Cancer Inst 1963;31:1333-56.

3 Ron E, Modan B, Boice JD. Mortality after radiotherapy for ringworm of the scalp. Am J Epidemiol 1988;127:713-25.

Muirhead CR, Kneale GW. Prenatal irradiation and childhood cancer. fournal of Radiological Protection 1989;9:209-12.

5 Harvey EB, Boice JD, Honeyman M, Flannery JT. Prenatal $x$ ray exposure and childhood cancer in twins. $N$ Engl f Med 1985;312:541-5.

6 Gardner MJ, Winter PD. Mortality in Cumberland during 1959-78 with reference to cancer in young people around Windscale. Lancet 1984;:216-7.

Teferen northern Scotland. Lancet 1986;i:266,385.

8 Committee on the Biological Effects of Ionising Radiation (BEIR V). Health effects of exposure to low levels of ionising radiation. Washington, DC: National Academy Press, 1990

9 Commission of the European Communities. Feasibility of studies on health effects in western Europe due to the reactor accident at Chernobyl and recommendations for research. Brussels: Commission of the European Communities, 1990. (Report EUR $12551 \mathrm{EN}$.)

10 Gardner MJ, Snee MP, Hall AJ, Powell CA, Downes S, Terrell JD. Results of case-control study of leukaemia and lymphoma among young people near Sellafield nuclear plant in West Cumbria. BMf 1990;300:423-9.

11 Darby SC, Doll R. Fallout, radiation doses near Dounreay, and childhood leukaemia. BMF 1987;294:603-7.

12 Dionian J, Wan SL, Wrixon AD. Radiation doses to members of the public around $A W R E$, Aldermaston, ROF, Burghfield, and AERE, Harwell. London: HMSO, 1987. (NRPB-R202.)

13 Payne CD, ed. The GLIM system. Release 3.77. Oxford: Numerical Algorithms Group Ltd, 1986.

14 Darby SC, Doll R, Gill SK, Smith PG. Long term mortality after a single treatment course with $x$ rays in patients treated for ankylosing spondylitis. treatment course with $x$ rays

15 United Nations Scientific Committee on the Effects of Atomic Radiation. Reports to the General Assembly. New York: United Nations, 1962, 1964, $1966,1969,1972,1988$

16 Stevens W, Thomas DC, Lyon JL, Till JE, Kerber RA, Simon SL, et al. Leukaemia in Utah and radioactive fallout from the Nevada test site. JAMA 1990;264:585-91.

17 Etherington G, Dorrian M-D. Radiocaesium levels, intakes and consequent doses in a group of adults living in southern England. In: Environmental contamination following a major nuclear accident. Vol 2. Vienna: International Atomic Energy Agency, 1990:327-38.

18 Morrey M, Brown J, William JA, Crick MJ, Simmonds JR, Hill MD. A preliminary assessment of the radiological impact of the Chernobyl reactor prelimin ass accident on the population of the European Community. Brussels: Con

9 National Radiological Protection Board. Committed doses to selected organs and committed effective doses from the intakes of radionuclides. London: HMSO, 1987. (NRBP-GS7.)

20 National Radiological Protection Board. Revised generalised derived levels for radioisotopes of strontium, iodine, caesium, plutonium, americium and curium. London: HMSO, 1987. (NRPB-GS8.)

(Accepted 18 February 1992) 